\title{
Flow of a yield stress fluid over a rotating surface
}

\author{
H. Tabuteau • J. C. Baudez $\cdot$ X. Chateau $\cdot$ P. Coussot
}

Received: 9 February 2006 / Accepted: 5 July 2006 / Published online: 15 November 2006

(C) Springer-Verlag 2006

\begin{abstract}
We study the flow of yield stress fluids over a rotating surface when both the viscoelastic solid behavior below a critical deformation $\left(\gamma_{c}\right)$ and liquid properties beyond $\gamma_{c}$ can play a significant role. We review the detailed characteristics of the flow in the solid regime in the specific case of a pure elongational strain (large height to radius ratio). We, in particular, show that there exists a critical rotation velocity $\left(\omega_{\mathrm{c}}\right)$ associated with the transition from the solid to the liquid regime. We then consider the specific case of lubricational regime (small height to radius ratio) in the liquid regime. In that case we describe the different possible evolutions of the equilibrium shape of the material as a function of the rotation velocity $(\omega)$, from which we extrapolate the transient shape evolutions as $\omega$ increases. We show that for a sufficiently large rotation velocity the sample separates into two parts, one remaining at rest around the rotation axis, the other going on moving radially. These predictions are then compared with systematic spin-coating tests under increasing rotation velocity ramps followed by a plateau at $\omega_{\mathrm{f}}$ with typical yield stress fluids. It appears that there exists a critical velocity below which the material undergoes a limited elongation and beyond which it starts to spread significantly over the solid surface. For a larger $\omega_{\mathrm{f}}$ value the sample forms a thick peripheral roll, leaving behind it a thin layer of fluid at rest relatively to the disc. These characteristics are in qualitative agreement with the theoretical predictions. Beyond a
\end{abstract}

H. Tabuteau $\cdot$ J. C. Baudez

Cemagref, Domaine des Palaquins,

Montoldre, France

X. Chateau $\cdot$ P. Coussot $(\square)$

LMSGC (UMR113 LCPC-ENPC-CNRS), Institut Navier,

Champs sur Marne, France

e-mail: Philippe.Coussot@lcpc.fr sufficiently large $\omega_{\mathrm{f}}$ value this roll eventually spreads radially in the form of thin fingers. Moreover, in agreement with the theory in the lubricational regime, the different curves of deformation vs $\omega$ fall along a master curve when the rotation velocity is scaled by $\omega_{\mathrm{c}}$ for different accelerations, different sample radii, or different material yield stress. The final thickness of the deposit seems to be mainly governed by the displacement of the roll, the characteristics of which take their origin in the initial stage of the spreading, including the solid-liquid transition.

Keywords Soft solids · Instability · Yield stress · Elongational flow

\section{Introduction}

To uniformly spread sewage sludges in fields, a technique is used which consists in dropping the material over a rotating disk (Thirion and Chabot 2003). The sludge, which is generally a yield stress fluid (Baudez and Coussot 2001), more or less widely spreads over the rotating surface and is thrown away in the field. Various effects such as flow, wall slip, or fracture may occur in that case but with the aim of understanding some basic properties of these process, in this study we will focus on the flow characteristics of a given amount of material flowing as a result of disk rotation and in the absence of wall slip or fracture.

This problem is reminiscent of spin coating, a process by which a fluid is spread over a rotating surface as a result of centrifugal force. In many cases the material is coated in the form of a solution from which the solvent evaporates (Birnie 1997), leaving a solid layer over the surface. The spin coating of paints, varnishes, or asphalts has the interest to produce almost uniform films. This technique is also 
widely used in the manufacture of integrated circuits (Huang and Chou 2003), magnetic and optical disks for data storage, color television screens, and optical mirrors. Most previous studies have focused on Newtonian fluids. It was particularly shown that a fluid layer of arbitrary initial shape tends to smooth and ultimately form a uniform film (Emslie et al. 1958). The process of thinning in time was then studied with the help of asymptotic methods (Higgins 1986). It was nevertheless shown that for slightly nonNewtonian fluids, namely, power-law fluids with a power smaller than 1, a uniform film cannot, in general, be obtained (Acrivos et al. 1960) because the viscosity is unbounded at low shear rates. Further studies of the spin coating of such fluids were then provided (Lawrence and Zhou 1991; Jenekhe and Schuldt 1984). From a general point of view experimental data are scarce so that existing theories can hardly be compared with relevant data.

Yield stress fluids are strongly non-Newtonian fluids in the sense that they a priori can flow only for stresses larger than a critical value. The spin coating of yield stress fluids was mainly the subject of theoretical studies. The authors (Jenekhe and Schuldt 1985; Burgess and Wilson 1996; Tsamopoulos et al. 1996) essentially addressed the difference from Newtonian films, particularly demonstrating that the fluid thickness cannot be uniform. Jenekhe and Schuldt (1985) first analyzed the spin-coating flow of yield stress fluids within the frame of the lubrication approximation. They showed that there is a fluid region close to the solid surface and an unsheared region close to the free surface, the thickness of which decreases with the distance from the central axis. In this context the pseudo steady-state flow profile, for which the unsheared region occupies the whole sample, is a rectangular hyperbolic profile (thickness $\propto 1 /$ distance from axis) centered at the origin. They also computed the profiles in time the shape of which is somewhat similar to the steady-state profile and progressively tend to it. Later Burgess and Wilson (1996) pointed out that the nonuniform radial flow of the unsheared region conflicts with the assumption of solid behavior in this region, an effect already noticed from a more general point of view by Lipscomb and Denn (1984) who showed that true plug flows can take place only within uniform flows of yield stress fluids. The authors overcame this conflict by using a biviscosity model and a suitable limiting process. Lastly, a systematic study of the spin coating of viscoplastic fluids was carried out by Tsamopoulos et al. (1996); they compared the flow characteristics from numerical simulations using a continuous viscosity function for representing the Bingham model and with different values of the Reynolds, Bingham, Bond, and capillary dimensionless numbers. However, because these numbers involve the socalled plastic viscosity the results cannot be easily compared with practical situations for which we generally have a relatively clear information concerning the material yield stress, but not concerning its detailed behavior (viscosity) in the liquid regime.

Also, these studies mainly addressed the well-developed flow of a yield stress fluid over a rotating disk but did not focus on the start and stoppage stages of the flow. However, it is precisely during these stages that the behavior of a yield stress fluid plays a critical role. Due to its stress yield a finite volume can be initially at rest and in its solid "state", the flow start implies that it undergoes a transition from its solid to its liquid state. It is likely that the flow characteristics at the solid-liquid transition depend, among others, on the material behavior in the solid regime. After some spreading, at a given distance the material should stop flowing when the centrifugal force, which is proportional to the fluid thickness, becomes smaller than the force needed for fluid flow. This property has already been identified as a basic means for estimating the yield stress of given volumes of yield stress fluids slumping or spreading over a fixed horizontal or inclined surface (Pashias et al. 1996; Coussot et al. 1996; Liu and Mei 1989; Balmforth et al. 2002). In our case the pressure gradient resulting from gravity effects (which adds to that due to inertia) plays a fundamental role in the equilibrium shape of the free surface. This pressure gradient was nevertheless neglected in the basic approach of spin coating by Emslie and subsequent approaches for yield stress fluids (Jenekhe and Schuldt 1985; Burgess and Wilson 1996; Tsamopoulos et al. 1996), which precludes relevant description of the asymptotic shape at stoppage.

In this study, we focus on the typical situations for which the yielding properties of the material play a major role: start flow then stoppage after some flow on a rotating disk. We first describe the constitutive equation of the material, which includes a solid regime and liquid regime ("Constitutive equation of pastes"). Then we consider the flow problem from a theoretical point of view ("Flow modeling") in simple cases by considering the solid and the liquid regimes as purely elongational and lubricational flows, respectively. Afterward, we present the results of systematic experiments with a typical yield stress fluid ("Experiments"). Lastly, we discuss these data with regard to the theoretical predictions ("Discussion and comparison between experiments and theory").

\section{Constitutive equation of pastes}

Pasty materials are generally considered as viscoelastic solids in their solid regime and yield stress fluids in their liquid regime. For the sake of simplicity, in this study, we assume that their behavior in the solid regime follows a simple Kelvin-Voigt model, which, in the context of small deformations, may be expressed as follows in terms of the 
relation between the strain and the stress tensors, namely, $\mathbf{E}$ and $\boldsymbol{\Sigma}$ :

$\boldsymbol{\Sigma}-\boldsymbol{\Sigma}_{0}=-p \mathbf{I}+F(\mathbf{E})=-p \mathbf{I}+2 G \mathbf{E}+2 \mu \dot{\mathbf{E}}$

in which $\mu$ is the viscosity and $G$ the elastic modulus of the material in the solid regime. In Eq. (1) we assumed that at the initial time the material is at rest and its stress distribution is $\boldsymbol{\Sigma}_{0}$. In the following we will also use the expression for the extrastress tensor defined as $\mathbf{T}=\boldsymbol{\Sigma}+p \mathbf{I}$, such that $\operatorname{tr} \mathbf{T}=0, p$ being the pressure, and $\mathbf{I}$ the unit tensor; and we will write the components of $\mathbf{T}$ as $\tau_{r r}, \tau_{r \theta}, \ldots$

Such a material is capable of getting out of its solid regime and flow like a liquid if the applied stress is sufficiently large. From a microstructural point of view the transition from the solid to the liquid state occurs when some network of interactions between the components of the material break. Thus, the transition from the solid to the liquid regime is basically associated with a critical deformation (Baudez and Coussot 2004; Uhlherr et al. 2005) from its initial state. Consequently, the state of reference of the material to be used for such a criterion is of critical importance. In practice this will be the state that will be reached after material set up, a state that will be necessarily reached after some flow in the liquid regime. In this study, we will assume that the material in its liquid state can reach back to its solid state as soon as it remains at rest for a short time (say, of the order of $1 \mathrm{~s}$ ). This assumption is in agreement with usual observations of flows of simple yield stress fluids in rheometrical tests. This particularly means that we neglect thixotropic effects that tend to increase the apparent yield stress of a pasty material left at rest.

Finally, we consider that the material reaches its liquid regime when the deformation undergone from its initial "solid" state (reached after a short time of rest) is such that:

$\sqrt{-E_{I I}}>\frac{\gamma_{c}}{2}$

in which $E_{I I}=-\operatorname{tr}(E)^{2} / 2$ is the second invariant of the strain tensor. Note that usual descriptions of the solid liquid transition for yield stress fluids assume that it occurs when the stress tensor is such that $\sqrt{-T_{I I}}>\tau_{c}$ in which $T_{I I}$ is the second invariant of the extrastress tensor $\mathbf{T}$ and $\tau_{\mathrm{c}}$ is the material yield stress (Oldroyd 1947). However, although such approach may be sufficient when a perfectly rigid behavior is assumed in the solid regime, in the general case this does not make it possible to ensure continuity between the two regimes that would be acceptable from a physical point of view. For example, for a viscoelastic material in the solid regime the stress reached for the critical strain depends on the flow history (e.g., a rapid deformation leads to a larger stress for a given strain than a slow deformation). In our case the yield stress finds a natural definition: it is the minimum value of $\sqrt{-T_{I I}}$ for which the critical strain may be reached, i.e., for an infinitely slow flow rate $(\dot{\mathbf{E}} \rightarrow 0)$, so that we obtain:

$\tau_{c}=\sqrt{-T_{I I}}=G \gamma_{c}$

In the liquid regime we assume that in steady state the material behavior is well represented by a model of the form:

$\boldsymbol{\Sigma}=-p \mathbf{I}+f(\mathbf{D})+\tau_{c} \frac{\mathbf{D}}{\sqrt{-D_{I I}}}$

in which $D_{I I}=-t r D^{2} / 2$ is the second invariant of the strain rate tensor and $f$ is a function such that $f \rightarrow 0$ when $D_{I I} \rightarrow 0$. A constitutive equation of the general form (Eq. 4) makes it possible to recover the usual Bingham, Casson, or Herschel-Bulkley models (Bird et al. 1982) by using specific forms of the function $f$. Note that in this study, again we neglect possible thixotropic effects, which induce apparent viscosity decrease during flow of pasty materials (Barnes 1997). The continuity between the behavior in the two regimes is ensured by the fact that the ultimate stress tensor in the material, which slowly stops flowing (i.e., $\mathbf{D} \rightarrow 0$ with negligible inertia effects), is such that $\sqrt{-T_{I I}}=\tau_{c}$.

\section{Flow modeling}

\section{Generalities}

The flow characteristics are found from the solution of the mass conservation and momentum equations, namely:

$\nabla \cdot \mathbf{u}=0$

$\rho \partial \mathbf{u} / \partial \mathbf{t}+\rho \nabla \mathbf{u} \cdot \mathbf{u}=\rho \mathbf{g}+\nabla \cdot \boldsymbol{\Sigma}$

here expressed in Eulerian coordinates, along with the constitutive Eqs. (1), (2), and (4) and the initial and boundary conditions. In Eqs. (5) and (6) $\mathbf{u}$ is the local velocity, $\rho$ the material density, and $\mathbf{g}$ the gravity. Here the main boundary condition takes its origin in the contact of the material with the solid surface of the disk. The initial conditions are given by the state of the material at the end of its setup (see below).

The general solution of such a problem can hardly be found explicitly. In this study, we will only consider specific, simple situations. Flow history may play a significant role on the characteristics of viscoelastic fluid flows, and a detailed description of the different flow stages is required for a relevant description of the flow. We assume that the material is first set up at rest on the solid surface (cf. "Material setup"). In a second stage the solid surface is 
rotated at a velocity, which possibly increases in time: $\omega(t)$. The material, which undergoes an additional (centrifugal) force, first slightly deforms in its solid regime (cf. "Solid regime") then, beyond a critical time (associated to a sufficiently large deformation), falls in its liquid regime and spreads more or less widely over the solid plane (cf. "Flow in the liquid regime").

\section{Material setup}

In the first stage the material, which was prepared in a given way, generally by some rapid mixing during which it reached its liquid state, is poured or set over the solid surface (which is initially at rest) in a mold. During this stage it flows in its liquid regime and eventually reaches some initial equilibrium state under the action of gravity. As soon as the fluid remains at rest a short time in this new position it falls in its solid regime and reaches a stress distribution $\boldsymbol{\Sigma}_{0}$ such that:

$0=\nabla \cdot \boldsymbol{\Sigma}_{0}+\rho \mathbf{g}$

Let us consider the specific case of a sample of vertical length much larger than its horizontal size. In that case the stress variations in the radial direction are negligible compared to those in the vertical direction so that we deduce from Eq. (7):

$$
\begin{aligned}
\boldsymbol{\Sigma}_{0}=-\rho g(h-z) \mathbf{e}_{z z}= & -\frac{\rho g(h-z)}{3} \mathbf{I} \\
& +\frac{\rho g(h-z)}{3}\left(\mathbf{e}_{\mathbf{r r}}+\mathbf{e}_{\theta \theta}-\mathbf{2}_{\mathbf{z z}}\right)
\end{aligned}
$$

in which $h$ is the height of the sample and $z$ is a current vertical level (see Fig. 1). The second term of the right hand side is the extrastress tensor. For slow motions the flow stops or starts for the critical height $z_{\mathrm{c}}$ at which the Von Mises criterion (Eq. 3) is reached which, taking into account Eq. (8), leads to $\rho g\left(h-z_{c}\right) / \sqrt{3}=\tau_{c}$. As a consequence, if the mold is removed without perturbing the sample, the critical depth below which the material remains in its solid regime writes:

$h_{c}=\frac{\sqrt{3 \tau_{c}}}{\rho g}$

Solid regime

\section{General case}

Now the material, which is initially in the state reached at the end of the set up (see "Material setup"), starts to flow as a result of the rotation of the solid surface. From Eqs. (1) and (7) we deduce that in the solid regime the momentum Eq. (6) here reduces to:

$\rho \frac{\partial \mathbf{u}}{\partial t}+\rho \nabla \mathbf{u} \cdot \mathbf{u}=-\nabla p+\nabla \cdot F$

We deduce that the flow characteristics only depend on the material behavior, the centrifugal force due to the rotation of the plate, and the boundary conditions, but gravity effects do not play any role. This implies that the sample should flow more or less in the same way at different depths. In the specific case of large $h / R$ values in which $R$ is the outer radius of the sample, edge effects (at the approach of the top or the bottom of the sample) are negligible and one can predict exactly the flow characteristics (see below). This ideal case (pure elongational deformation) may be used as a reference to get a qualitative idea of the flow characteristics in the general case. Because gravity effects are always absent the solution of momentum equation in this ideal case may be extrapolated to situations for which $h / R$ is of the order of 1 when edge effects may start to play a significant role.

\section{Elongational deformation in the solid regime}

We describe the flow characteristics in the static cylindrical coordinate frame (r, $\theta$, and $z$ ) (cf. Fig. 1). Here, we assume that the material has an approximately cylindrical initial shape and that $h_{0} \gg r_{0}$ in which $h_{0}$ and $r_{0}$ are the initial cylinder height and radius. In that case edge effects can be neglected in most of the material, which flows under the action of centrifugal force only, a force which does not depend on $z$. As a consequence all occurs as if the sample was submitted to an increased (radial) gravity, which should consequently tend to squeeze it vertically and homogeneously. More precisely, the flow is similar in any fluid portion so that $v_{r}$ does not depend on $z$. In addition, if the fluid velocity in the vertical direction $\left(v_{z}\right)$ depended on $r$ the cylindrical layers of materials at different distances would move relatively to each other in some specific direction, which is particularly not compatible with the boundary

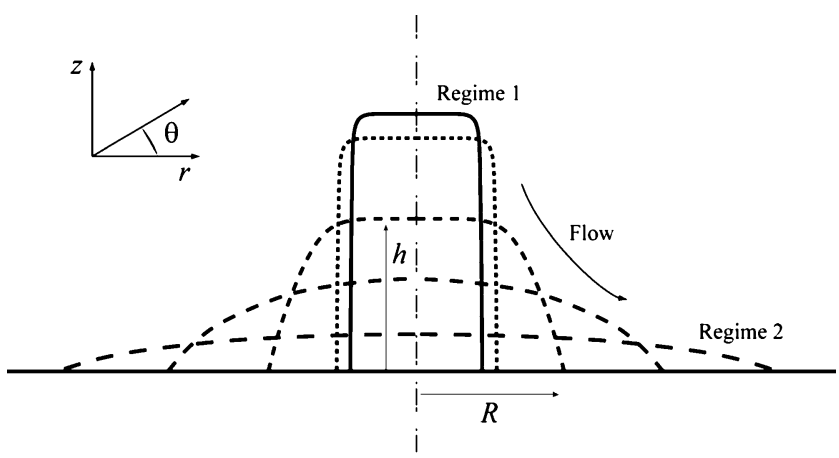

Fig. 1 Aspects of the cross-section of a pasty material at different times during spin-coating process 
condition (vertical velocity equal to zero along the solid plate because the material remains in contact with it). At last the flow is symmetrical by rotation around the axis so that the variables do not depend on $\theta$, and if transient effects are negligible the tangential velocity, is equal to that of the disk, i.e., $v_{\theta}=\omega r$.

Because $v_{r}$ and $v_{z}$ are related by the mass conservation (Eq. 5, which takes the form of $\frac{1}{r} \frac{\partial\left(r v_{r}\right)}{\partial r}+\frac{\partial v_{z}}{\partial z}=0$ here), we deduce that the velocity field takes the following form:

$v_{r}=\frac{a}{2} r ; \quad v_{\theta}=\omega r ; \quad v_{z}=-a z$

in which $a$ is the strain rate which only depends on time $(t)$. Such a velocity field corresponds to a uniaxial stretching for which the sample keeps a cylindrical shape. From boundary conditions we get $a=2 R / R$. In the limit of small deformations the strain tensor may be computed from Eq. (11): $\mathbf{E}=\left(\int_{0}^{t} a d t\right)\left[-\mathbf{e}_{\mathbf{z z}}+\left(\mathbf{e}_{\mathbf{r r}}+\mathbf{e}_{\theta \theta} / 2\right]\right.$. We deduce that the deformation is homogeneous in the sample and, from Eq. (1), that the stress tensor only depends on time with $\tau_{r r}=\tau_{\theta \theta}=-\tau_{z z} / 2$ and $\tau_{r \theta}=\tau_{r z}=\tau_{\theta z}=0$.

Consequently, if $a \ll \omega$ and $\partial a / \partial t \ll \omega^{2}$ (negligible inertia effects) the momentum equation (Eq. 6) at the first order reduces to:

$-\rho \omega^{2} r=-\frac{\partial p}{\partial r}$

which may be solved in:

$p(r)=p(0)+\rho \omega^{2} \frac{r^{2}}{2}$

When $\omega=0$ the pressure $(p(r)=p(0))$ is equal to the ambient pressure $p_{0}$ (because gravity effects are negligible). When $\omega$ differs from zero the sample region along the central axis is unaffected by the rotation and we can reasonably assume that $p(0)$ remains equal to $p_{0}$. Using the boundary condition at the periphery $(r=R): \boldsymbol{\Sigma} \cdot \mathbf{n}=\left(-p+\tau_{r r}\right) \mathbf{n}=-p_{0} \mathbf{n}$, we find from Eq. (1):

$\mu a+G \int_{0}^{t} a d t=\rho \omega^{2} \frac{R^{2}}{2}$

In the solid regime the variations of the radius $R$ are negligible compared to those resulting from the increase in rotation velocity, so that we can take $R=r_{0}$ in the right hand side of Eq. (14). The maximum error due to this approximation is $\Delta r=\left(r_{c}-r_{0}\right) / r_{0}$ in which $r_{\mathrm{c}}$ is the critical radius when the liquid regime is reached, i.e., for the critical deformation. Remarking that $\int_{0}^{t} a d t=2 \ln \left(R / r_{0}\right)$ it follows from Eq. (2) that $2 \ln \left(r_{c} / r_{0}\right)=\gamma_{c} / \sqrt{3}$. Because $\gamma_{c}$ is typically of the order of 0.1 [16], we find that $\Delta R$ is of the order of $3 \%$.
Let us consider that at the initial time we impose a rotation velocity $\omega$ to the disk initially at rest. In that case the solution of Eq. (12) writes:

$R=r_{0} \exp \left[\frac{\rho \omega^{2} r_{0}^{2}}{4 G}(1-\exp (-G t / \mu))\right]$

Over long times $(t>\mu / G)$ the deformation reaches its maximum value when $R=r_{\mathrm{m}}$ such that $\ln \left(r_{m} / r_{0}\right)=$ $\rho \omega^{2} r_{0}^{2} / 4 G$. Taking into account Eq. (2) we find the critical velocity for which the critical deformation is ultimately reached (see above), i.e., such that $r_{\mathrm{m}}=r_{\mathrm{c}}$ :

$\omega_{c}=\sqrt{\frac{2 \tau_{c}}{\sqrt{3} \rho r_{0}^{2}}}$

Thus, for rotation velocity $\omega$ smaller than $\omega_{\mathrm{c}}$ the critical deformation is never reached, i.e., the material remains in its solid regime. For $\omega=\omega_{\mathrm{c}}$ the critical deformation is reached after an infinite time. For rotation velocity $\omega$ larger than $\omega_{\mathrm{c}}$ the critical deformation is reached at the (finite) time:

$t_{c}=-\frac{\mu}{G} \ln \left[1-\left(\frac{\omega_{c}}{\omega}\right)^{2}\right]$

When the rotation velocity increases linearly in time, i.e., $\omega=k t$, we find by integration of Eq. (14):

$R=r_{0} \exp \frac{\rho k^{2} r_{0}^{2}}{4 G}\left[\frac{\mu^{2}}{G^{2}}(1-\exp (-G t / \mu))+\left(t^{2}-\frac{2 \mu t}{G}\right)\right]$

For large acceleration the critical time at which the critical radius is reached is small, namely, $G t_{\mathrm{c}} / \mu \ll 1$, and we deduce from Eq. (18) that $t_{c} \approx\left(2 \mu \gamma_{c} / \sqrt{3} \rho k^{2} r_{0}^{2}\right)^{1 / 3}$. For sufficiently low acceleration the critical time is large $\left(G t_{\mathrm{c}} / \mu \gg 1\right)$ and we have $t_{c} \approx\left(2 G \gamma_{c} / \sqrt{3} \rho k^{2} r_{0}^{2}\right)^{1 / 2}$ from which we deduce that the critical velocity is again given by Eq. (16).

Flow in the liquid regime

\section{Surface tension effects}

As the fluid spreads over the solid surface it is expected that this mainly occurs via the shear due to the relative motion of the fluid and the solid surface, but surface tension effects may also become significant. Let us compare the viscous energy loss $\left(d W_{\mathrm{v}}\right)$ to the surface energy change $\left(d W_{\mathrm{s}}\right)$ during an elementary spreading (say of $d R$ ) over a solid surface of a horizontal layer of uniform thickness much smaller than its radial extent $(h \ll R)$. The surface energy change is the sum of the changes in the interfacial energy between the solid and the ambient gas, the material and the ambient gas, and the solid and the material. These energies are proportional respectively to the changes in the surfaces of the solid-gas, the material-gas, and the solid-material interfaces, via 
coefficients equal to the interfacial tensions $\gamma_{\mathrm{SG}}, \gamma_{\mathrm{LG}}$, and $\gamma_{\mathrm{SL}}$, respectively, which are related by the Young's equation $\gamma_{S G}=\gamma_{L G} \cos \theta+\gamma_{S L}$ where $\theta$ is the wetting angle between the three phases. When the thickness of the layer is small the amplitude change in these surfaces is $2 \pi R d R$, from which we deduce $d W_{s} \approx 2 \pi R \gamma_{L G}(1-\cos \theta) d R$. Note that from natural considerations $\gamma_{\mathrm{LG}}$ can be considered as typically equal to the surface tension of water with air, i.e., $0.07 \mathrm{~Pa} \mathrm{~m}$. Assuming that the flow is mainly a simple shear flow in the radial direction the shear strain $(\gamma)$ at a distance $r$ is proportional to the shear strain at the periphery: $r d R / R h$, so that the total viscous energy loss writes $\iint_{\Omega} \tau \gamma d \omega=$ $\int_{0}^{R} \tau(r d R / R h)(2 \pi h r d r)$ in which $d \omega$ is a volume element. In the limit of slow flows $\left(\tau \approx \tau_{\mathrm{c}}\right)$ we find: $d W_{v} \approx$ $2 \pi R^{2} \tau_{c} d R / 3$. Surface tension effects remain negligible when the energy loss due to viscous dissipation is much larger than the surface energy change, i.e., when $d W_{\mathrm{v}} \gg d W_{\mathrm{s}}$, which leads to the condition:

$\tau_{c}>>\frac{3(1-\cos \theta) \gamma_{L G}}{R}$

\section{Lubricational flow in the liquid regime}

The description of flow characteristics from an analytical point of view is extremely difficult in the general case. In this study, we will consider the specific case when the layer thickness becomes sufficiently small compared to its radial extent $(h \ll r)$ (regime 2, see Fig. 1). We assume (long-wave or lubrication approximation) that, relative to the rotating surface, the main motion is in the radial direction $\left(v \ll v_{r}\right)$ and that the gradient of the velocity along the vertical direction is much larger than in the radial direction. It follows from the constitutive Eq. (4) that the only nonnegligible component of the extrastress tensor is $\tau_{r z}$ and, using again the assumptions for negligible inertia, the momentum equation reduces to:

$-\rho \omega^{2} r=-\frac{\partial p}{\partial r}+\frac{\partial \tau_{r z}}{\partial z}$

$0=-\rho g-\frac{\partial p}{\partial z}$

Taking into account the boundary condition along the free surface, i.e., $\boldsymbol{\Sigma} \cdot \mathbf{n}=-p_{0} \mathbf{n}$ for $z=h(r)$, we deduce from Eq. (20b) the pressure distribution:

$p=p_{0}+\rho g(h(r)-z)$

and from Eq. (20a) the shear stress distribution in the fluid:

$\tau_{r z}=\left(\rho \omega^{2} r-\rho g \frac{\partial h}{\partial r}\right)(h-z)$
In the specific case of a sample of cylindrical shape with a horizontal top free surface $(\partial h / \partial r=0)$ we can deduce from Eq. (22) that a flow in the liquid regime should start as soon as the maximum shear stress [namely, $\tau_{r z}(0)$ ] at the periphery $\left(r=r_{0}\right)$ is larger than the yield stress. There is therefore a critical rotation velocity associated with flow start in the lubricational regime under such conditions:

$\omega_{c}^{*}=\sqrt{\frac{\tau_{c}}{\rho r_{0} h_{0}}}$

This expression obviously differs from the critical velocity (Eq. 16) associated to the solid-liquid transition under the elongational assumption because the underlying assumptions are different $\left(h_{0} / r_{0} \ll 1\right.$ in the former case and $h_{0} / r_{0} \gg 1$ in the latter case). However, it is worth noting that expressions 16 and 23 provide very close values for intermediate aspect ratios, say $h_{0} / r_{0}$ of the order of 1 , which suggests that these expressions might be valid up to such aspect ratios, thus ensuring a continuity of the critical velocity between the two asymptotic regimes.

More generally, let us now assume that the whole material flowed at least during a short time in its liquid regime then stopped flowing. In that case the maximum shear stress at each distance from the central axis, i.e., that reached along the disk, must be equal to the yield stress $\left(\sqrt{-T_{I I}}=\tau_{r z}(0)=\tau_{c}\right)$. Using the dimensionless variables $R=r / r_{0}, H=h / h_{0}$, and $\Omega=\omega / \omega_{c}^{*}$, we obtain from Eq. (22) the dimensionless form of the equation giving the equilibrium shape of the free surface in that case:

$\Omega^{2} R H-\alpha H H^{\prime}=1$

in which $\alpha=\rho g h_{0} / \tau_{c}$.

Two limiting cases can first be distinguished from Eq. (24). When $\Omega$ is small, or more precisely, when $\Omega^{2} R H^{\prime} 1$, which means that $\rho \omega^{2} r h / \tau_{\mathrm{c}} \ll 1$, i.e., the effect of rotation is negligible, Eq. (24) reduces to $-\alpha H H^{\prime}=1$, which is the equation for the ultimate shape at stoppage of a yield stress fluid, which spreads over a horizontal surface at rest (cf. Coussot et al. 1996); the solution of which writes:

$H=\sqrt{\left(H_{0}^{2}-2 R\right) / \alpha}$

Some typical shape of the fluid in that case is shown in Fig. 2 (this corresponds to $\Omega=0$ ). The maximum distance reached by the fluid, i.e., for $H=0$, is $R_{\max }=\left(H_{0}^{2} / 2\right.$, and the total volume of material may be expressed as:

$V=\frac{2 \pi}{15} H_{0}^{5 / 2}=\frac{8 \sqrt{2} \pi}{15} R_{\max }^{5 / 2}$

from which we see that the fluid extent may be predicted from its volume.

When $\Omega$ is sufficiently large, or more precisely when $\Omega^{2} R H \gg 1$, the right hand side of Eq. (24) can be neglected 


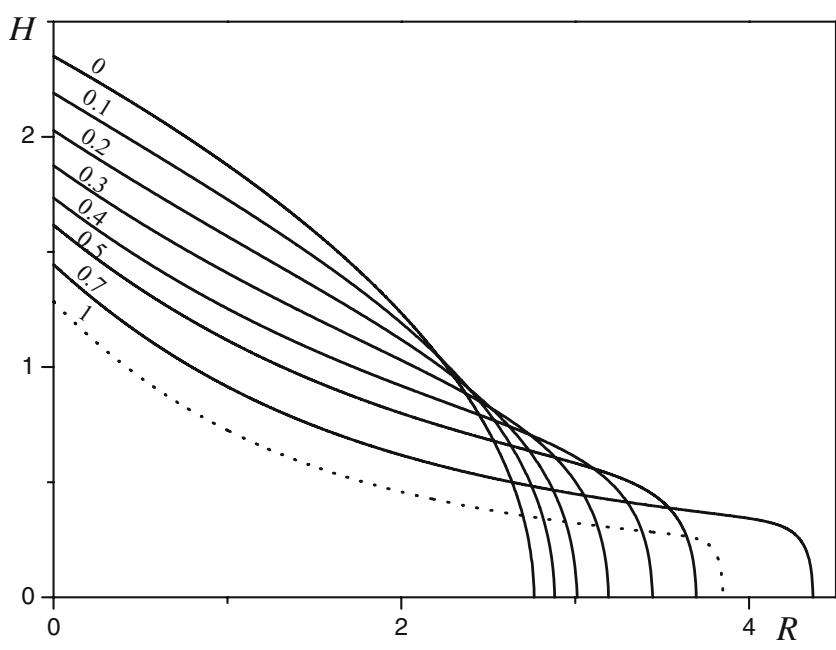

Fig. 2 Equilibrium free surface profiles under the lubrication assumption in the case $\alpha=1$ for a given dimensionless sample volume $V=\int 2 \pi H R d R=9$ and different values of $\Omega^{2}$. The dotted line corresponds to the first profile obtained beyond the critical value $\Omega_{c}^{2}$

and this equation reduces to $\Omega^{2} R=\alpha H^{\prime}$, the solution of which writes:

$H=H_{0}+\frac{\Omega^{2} R^{2}}{2 \alpha}$

Here, due to inertia effects, the material thickness increases with the distance from the center. A fluid stoppage would imply that $H=0$ at some finite distance, which is not possible. Fluid stoppage is possible only if there is a vertical rigid boundary keeping the material in place at some distance.

Let us now consider the general case. Equation (24) may be expressed in the form $\alpha H^{\prime}=\Omega^{2} R-1 / H$ (curve 1) from which we can draw a phase diagram giving the evolutions of $H^{\prime}$ as a function of $H$ as $R$ varies (cf. Fig. 3). In such a diagram the free surface profile is obtained from a path going through the different curves obtained for different increasing values of $R$. It is worth noting that $\alpha \partial H^{\prime} / \partial H=\Omega^{2} / H^{\prime}+1 / H^{2}$, from which we deduce that an important curve to be drawn in Fig. 3 is the curve 2: $H^{\prime}=-\Omega^{2} H^{2}$, which gives the position at which the slope of the profile reaches zero. For a fixed rotation velocity we deduce three possible evolutions of $H$ as a function of $R$ depending on the value of the central height $\left(H_{0}\right)$.

(A) For $H_{0}$ smaller than the critical value $H_{i}$ corresponding to the intersection between the initial curve 1 for $R=0$ and the curve $2, H^{\prime}$ continuously decreases toward minus infinity as $H$ tends to zero. The shape of the resulting profile is qualitatively similar to that obtained for a negligible rotation velocity.

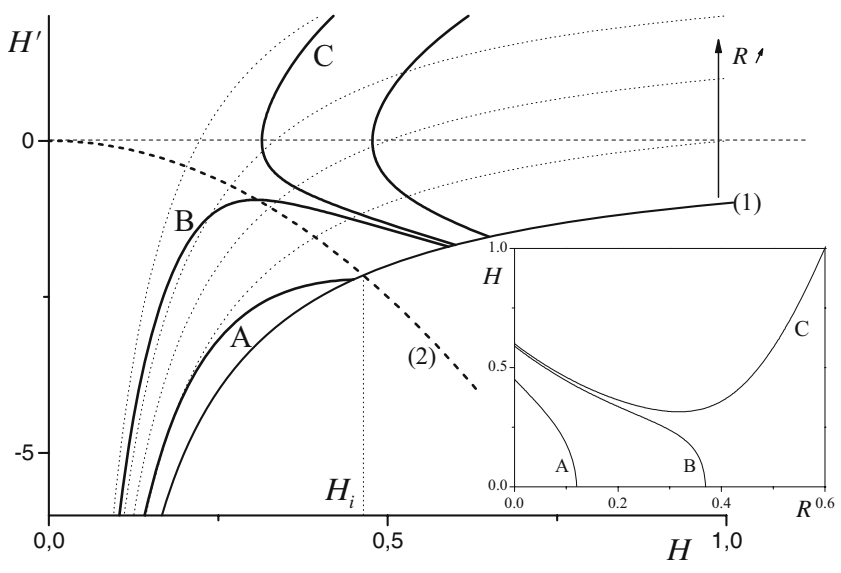

Fig. 3 Phase diagram for the sample height as a function of the distance under the lubrication assumption in the case $\alpha=1$ and $\Omega^{2}=10$. The thin dotted lines correspond to curve 1 (see text) at different finite distances $R$. The continuous thick lines are examples of the evolutions of $H^{\prime}$ as a function of $H$ along the corresponding equilibrium free surface profiles (see insert) for different values of the initial height

(B) For $H_{0}$ larger than $H_{i}$ but lower than a second critical value, $H^{\prime}$ first increases then reaches a maximum value (associated to the intersection with curve 2) and finally decreases toward minus infinity as $H$ tends to zero.

(C) For $H_{0}$ larger than the second critical value, the curve $H^{\prime}(H)$ increases and reaches the abscissa axis with an infinite slope, so that $H^{\prime}$ becomes positive and the height then increases and tends to infinity.

Only the cases (A) and (B) correspond to equilibrium profiles.

An equivalent analysis with now a given central height $H_{0}$ and different velocities can be made from this phase diagram: This is now the curve 1 , which moves toward the largest $H$ as the rotation velocity increases, so that $H_{i}$ increases. As a consequence a shape of the type A is obtained for a rotation below a critical value, a shape of the type B for a rotation velocity larger than this value but lower than another critical value and a shape of the type $\mathrm{C}$ is obtained for a rotation velocity larger than this second critical value. A typical set of shapes A and B obtained for different rotation velocities is shown in Fig. 2.

Until now we considered the expected shapes of the free surface profiles independently of the volume of material. This is a limiting factor. Let us consider a sample of volume $V_{0}$; for a relatively small rotation velocity, one can find one and only one equilibrium shape associated to $V_{0}$, from which we deduce the central height $H_{0}$; for rotation velocities larger than a critical value there is no equilibrium shape associated to $V_{0}$. This is illustrated from the example of Fig. 2 in which the (dimensionless) critical velocity beyond which there is no equilibrium shape (for a given sample volume) is equal to 1 . 


\section{Transient flows}

We consider a sample of given cylindrical shape initially at rest over the solid plate. Then one applies a given rotation velocity to the disk at the initial time. If the central height is larger than that associated to the equilibrium profile (if it exists) the material spreads over the disk and its central height decreases. Otherwise there might be some partial deformation at least in a peripheral region because the cylindrical shape does not correspond to any equilibrium profile, but we will not study that case further. As long as the central height is larger than that associated to the equilibrium profile for this sample volume the equilibrium shape predicted by Eq. (24) from this central height corresponds to a larger volume. We can nevertheless compute some "theoretical equilibrium profile" from Eq. (24) by using the limiting condition concerning the sample volume by truncating the equilibrium profile for this central height at an appropriate distance (see Figs. 4 and 5). Now if we assume that as the material spreads over the disk, the instantaneous profile corresponds to this theoretical equilibrium profile we get an approximate picture of the successive profiles in time.

Two cases can then be distinguished. If the rotation velocity is smaller than a critical value (see above) there is an equilibrium shape associated to the sample volume and the central height eventually reaches its critical value associated to this equilibrium shape, so that the fluid stops flowing (see Fig. 4). If the rotation velocity is larger than the critical value the fluid spreads and as it decreases the central height reaches a value for which there exists an equilibrium shape for the critical rotation velocity but for a smaller material volume, so that some central region of the

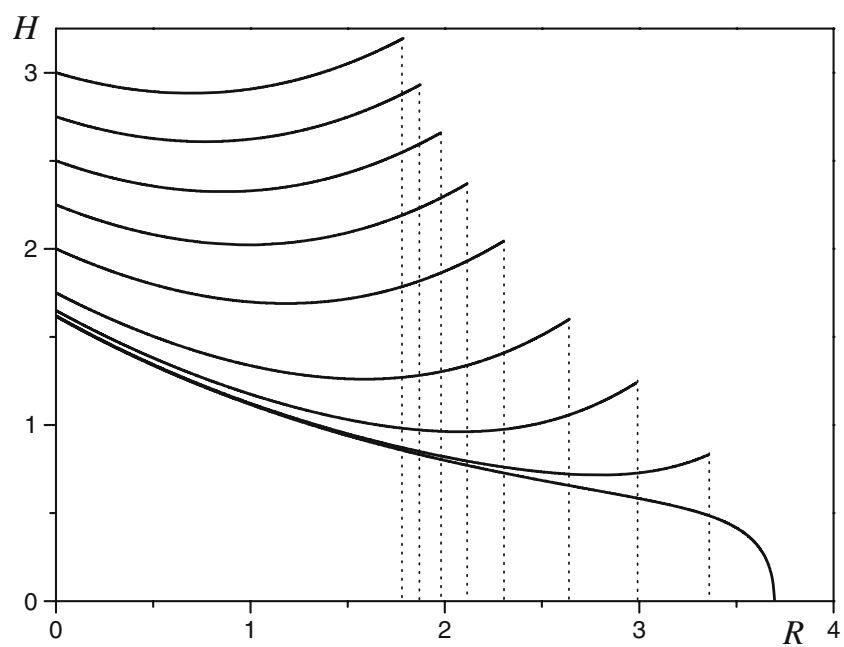

Fig. 4 Theoretical free surface profiles according to the equilibrium equation for a limited dimensionless volume $(V=9), \Omega^{2}=0.5$, and different central heights. The dotted lines correspond to the virtual boundary. The bottom line is the equilibrium profile

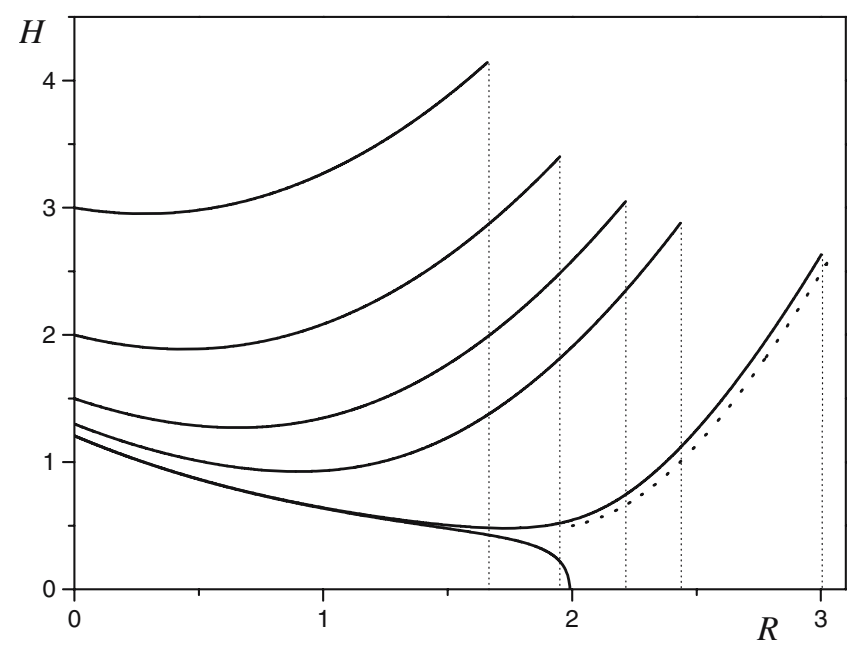

Fig. 5 Theoretical free surface profiles according to the equilibrium equation for a limited dimensionless volume $(V=9), \Omega^{2}=1.2$, and different central heights. The dotted lines correspond to the virtual boundary. Below a critical central height one obtains an equilibrium profile (bottom line) but the dimensionless volume is smaller than 9 in that case

fluid tends to stop, while the rest of the fluid takes an indeterminate shape, which might resemble that obtained for a slightly larger central height, and the fluid in this region goes on flowing farther (see Fig. 5). Thus, we expect some separation of the fluid into two parts. To figure out what might occur in that case we have drawn the profile as it is predicted by Eq. (24) starting from an arbitrary thickness at a distance equal to the maximum radius reached by the equilibrium curve part. We can expect that this series of profiles gives an idea of the evolutions of the fluid shape in time.

\section{Experiments}

We carried out experimental tests with pasty materials. In the following we first describe the experimental setup and the materials, then we report the main observations.

Experimental setup and procedures

Our experimental setup consisted of a horizontal disk of a radius of $40 \mathrm{~cm}$ driven by a variable speed motor at a rotation frequency in the range $0.1-50 \mathrm{~Hz}$ ( 3 to $1,500 \mathrm{rpm})$ with frequency fluctuations less than $0.1 \mathrm{~Hz}( \pm 1.5 \mathrm{rpm})$. Vibrations produced by the motor were damped by a coupling with the disc via a system of ball bearings reducing the fluctuations around the horizontal. The disc made of stainless steel was covered with a smooth polymeric film (Venilia) except in some cases for which it will be mentioned. The rotation velocity of the disc could be increased linearly from rest to the largest velocity in less 
than $1 \mathrm{~s}$. We could check that inertia effects are negligible for this range of acceleration and the induced deformations.

We mainly used a commercial hair gel (Yplon S.A., Belgium), basically made of water and Carbopol, with the internal structure of a physical gel of density close to that of water. Previous studies showed that this material exhibits the rheological behavior of a typical yield stress fluid with a flow curve, which may well be represented by a Herschel-Bulkley model (Baudez et al. 2002). Here the yield stress, as determined from a series of creep tests at different levels with a parallel plate rheometer (see Baudez et al. 2002), was found to be $70 \mathrm{~Pa}$. To change the yield stress value we also used suspensions of Carbopol ${ }^{\circledR} 940$ (ACROS Organics, France) at different solid fractions, prepared according to the procedure described in Curran et al. (2002). For the range of yield stresses tested it may be checked that surface tension effects are negligible according to Eq. (19). The fraction of bubbles was reduced by prolongated mixing.

The experimental procedure was as follows: a hollow PVC cylinder was set at the center of the disc. Then the material was gently poured with a spoon into this mold so as to fill it, which made it possible to limit the formation of large bubbles between the gel and the solid. Then the cylinder was lifted leaving the material possibly spread on the disc under the action of its own weight. To best control the initial conditions of our spin-coating tests, we could define the largest initial sample height $\left(h_{0 \mathrm{~m}}\right)$ for which the sample apparently kept its cylindrical shape after lifting the cylinder. For the hair gel we found $h_{0 \mathrm{~m}}=12.3 \mathrm{~mm}$. For a larger height the material slightly spread and went on slowly flowing for several minutes but it basically kept a cylindrical shape (cf. Fig. 7a). It is worth noting that this value for $h_{0 \mathrm{~m}}$ almost exactly corresponds to the critical height (as given by Eq. 9) for which the sample should remain in its solid regime under the assumption of pure elongational flow, namely, $12.1 \mathrm{~mm}$ for the hair gel. Thus, for an initial height larger than $h_{0 \mathrm{~m}}$ the liquid regime is reached in some part of the sample, which tends to spread radially, but because the aspect ratio is not too large it is likely that most of the material flows in this regime. At the end of the flow the material reaches a new solid state of reference, from which we can start the spin-coating tests. For the main height used in our case $(20 \mathrm{~mm})$ we started the experiment (disc rotation) $3 \mathrm{~min}$ after lifting the cylinder, a time beyond which there was apparently no significant variation of the sample height and radius. For each run the rotation velocity of the disc was increased at a constant rate up to a particular value $\left(\omega_{\mathrm{f}}\right)$ at which it then remained constant. With such a procedure the time variations of the rotation velocity, and more precisely the acceleration stages, were well controlled, which was not always the case in previous works. After each run the gel sample was removed and another sample was set up, which particularly made it possible to avoid drying problems.

A high-speed video camera (250 frames/s) was set up either above or on the side of the disk to film the sample flow. The central part of the disc was lighted with a stroboscope $(1 / 50,000 \mathrm{~s})$. The camera was connected to a videotape recorder connected to a PC. By filming the sample from above we could measure the time evolutions of the average spreading in time, i.e., the average radius of the line of contact between the solid, the fluid, and the air: $\langle R(t)\rangle=(1 / 2 \pi) \int_{0}^{2 \pi} r(\theta, t)_{h=0} d \theta$, i.e., the maximum average sample radius. By filming the side of the sample we could measure the sample profile from the position of tracers (pearls of diameter $1.5 \mathrm{~mm}$ ) put onto the top free surface along radial directions (see Fig. 9).

\section{Experimental results}

\section{Generalities}

Let us consider the evolution of the spreading $(<R(t)>)$ in time for different final levels of rotation velocities with the same acceleration in the increasing stage. We chose to represent the data in the form of the apparent total strain $\left(\ln \left(<R>/ r_{0}\right)\right)$ as a function of time (Fig. 6). For a given acceleration, for any value of $\omega_{\mathrm{f}}$ the sample undergoes a rapid deformation during the increasing velocity period, and goes on spreading more slowly during the constant velocity period. This leads to a break in the spreading curve slope represented in a logarithmic scale. As a consequence the curves obtained for different $\omega_{\mathrm{f}}$ superimpose along a single curve in the increasing stage and depart from this curve as soon as the rotation velocity becomes constant (see Fig. 6).

Looking at the shape of the sample in time different regimes may be distinguished as a function of the value of $\omega_{\mathrm{f}}$. Below some critical value, $\bar{\omega}_{c}$ (about $145 \mathrm{rpm}$ for the hair gel), the sample height at different radial distances decreases uniformly, i.e., the sample profile remains horizontal and its sides remain approximately straight (see Fig. 7b) (regime 1). Thus, in this regime the material deforms as in a simple elongational flow. In this regime $<R(t)>$ only slightly increases during the run and over long times the total strain seems to tend to a finite, asymptotic value (about 0.12 for the hair gel). A new critical deformation $\left(\gamma_{0}\right.$, here equal to 0.035$)$ may be identified as the deformation reached when the spreading curve for $\bar{\omega}_{c}$ starts to depart from the master curve.

For $\omega_{f}>\bar{\omega}_{c}$ (regime 2), the sample deformation is similar to that observed for smaller final rotation velocities in a first stage; it resembles an elongational flow. When the rotation velocity overcomes $\bar{\omega}_{c}$ (during the disc acceleration) the sample shape starts to differ from that of a 


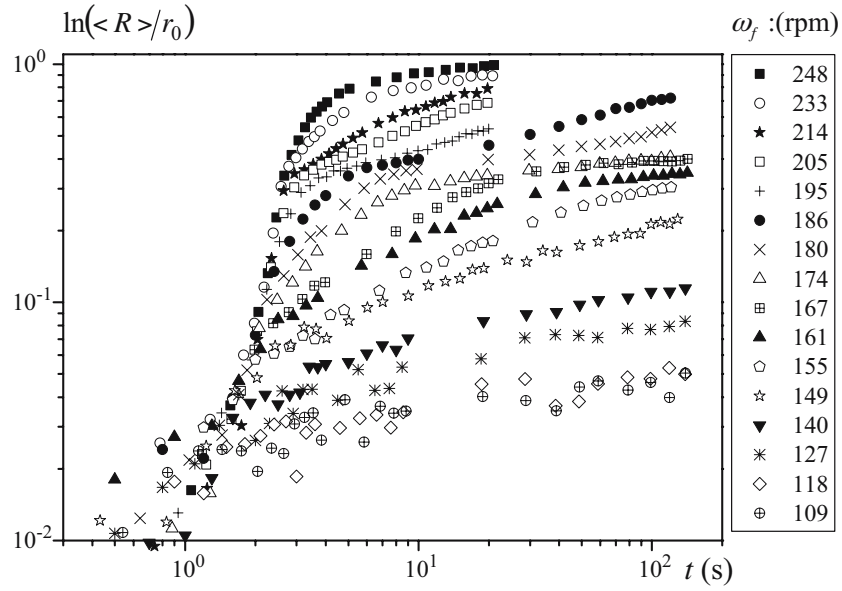

Fig. 6 Spreading as a function of time of a hair gel sample (initial dimensions: $r_{0}=37.4 \mathrm{~mm}$ and $h_{0}=18 \mathrm{~mm}$ ) for different ramps of rotation velocities: same acceleration $\left(k=7.7 \mathrm{rad} \mathrm{s}^{-2}\right)$ but different final levels of the rotation velocity $\left(\omega_{k}\right)$

cylinder: the periphery tends to collapse and flow outward radially, leaving a central part (dome) of larger height, but the edge remains straight (cf. Fig. 7c). For a larger final rotation velocity (regime 3 ) these effects are increased: the central dome no longer deforms while the outer part of the sample forms a roll, i.e., a short, circular region of larger depth, which rapidly spreads outward; thus, the sample tends to separate into two parts, one central static one and the other in the form of a rapidly moving outer roll (see Figs. $7 \mathrm{c}, 8$, and 9). This separation into two parts clearly appears from the observation of the displacement of the tracers at the top of the sample (see Fig. 10): the tracers close to the central part of the sample stop first while those farther from the center go on moving radially. A strong difference subsists during most of the spreading between the roll thickness $\left(h_{\mathrm{r}}\right)$ and the thickness $\left(h_{\mathrm{f}}\right)$ of the layer just behind it, for example for a sample of initial characteristics $r_{0}=37.4 \mathrm{~mm}$ and $h_{0}=13 \mathrm{~mm}$ and under the acceleration $k=7.72 \mathrm{rad} \mathrm{s}^{-2}$ we have: $\langle R>=74 \mathrm{~mm}(\omega=201 \mathrm{rpm})$, $h_{\mathrm{r}}=4.2 \mathrm{~mm}$, and $h_{\mathrm{f}}=1.68 \mathrm{~mm} ; \quad<R>=91.6 \mathrm{~mm}$ $(\omega=246 \mathrm{rpm}), h_{\mathrm{r}}=1.95 \mathrm{~mm}$, and $h_{\mathrm{f}}=0.58 \mathrm{~mm}$; and $<R>=105 \mathrm{~mm}(\omega=291 \mathrm{rpm}), h_{\mathrm{r}}=1.62 \mathrm{~mm}$, and $h_{\mathrm{f}}=0.5 \mathrm{~mm}$. For sufficiently large rotation velocities, the surface covered by the sample significantly increases and there is in a last stage an undulation of the roll, which grows in the form of fingers (see Fig. 8).

\section{Influence of the nature of the solid surface}

We carried out similar experiments with a disc now covered with either sand paper or commercial (hydrophobic) coating consisting mainly of Teflon ${ }^{\circledR}$ (BYTAC, roughness of the order of $1 \mu \mathrm{m})$. We observed that whatever the nature of the substrate, the sample significantly spreads beyond the same critical value $\omega_{c}$ and the end of the circular spreading is
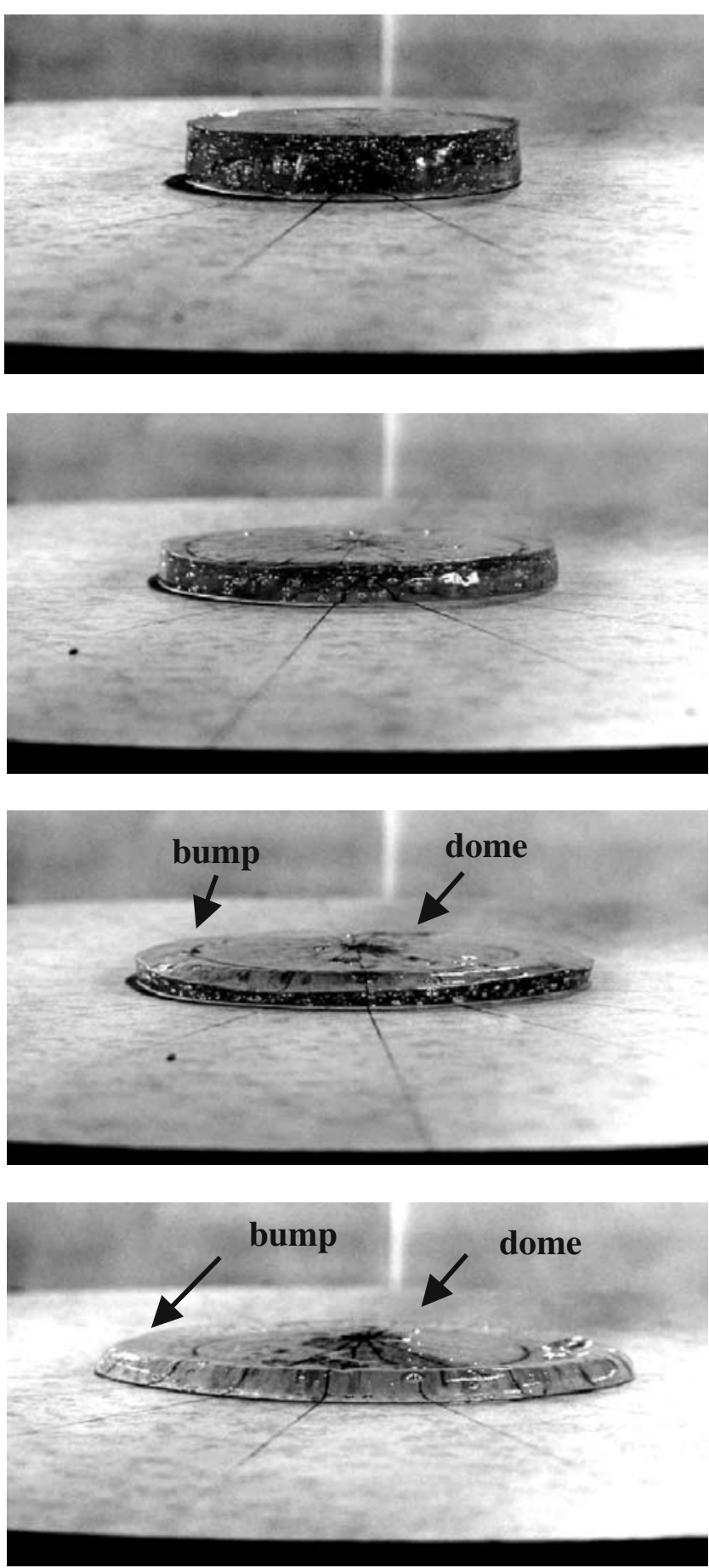

Fig. 7 Different aspects of the final shape of the sample for different values of the final rotation velocity for the hair gel $\left(r_{0}=37.4 \mathrm{~mm}\right.$, $h_{0}=13 \mathrm{~mm}$, and $k=7.72 \mathrm{rad} . \mathrm{s}^{-2}$ ): a cylindrical sample at rest before the test; b $\omega_{\mathrm{f}}=141 \mathrm{rpm}$ : elongation (regime 1); $\mathbf{c} \omega_{\mathrm{f}}=161 \mathrm{rpm}$ : dome and roll formation (regime 2); and $\mathbf{d} \omega_{\mathrm{f}}=214 \mathrm{rpm}$ : separation into two parts (regime 3)

obtained almost for the same value of the average radius. Moreover, the evolutions of the spreading in time for the different surfaces for $\omega_{\mathrm{f}}>\omega_{\mathrm{c}}$ appear rather similar (see Fig. 11). This means that possible wall slip or capillary forces play a negligible role in the process. 
Fig. 8 Different aspects (view from upper) of the spreading for different final rotation velocities for the hair gel of Fig. 7 in regime 3: a $\omega_{\mathrm{f}}=206 \mathrm{rpm}$; b $\omega_{\mathrm{f}}=251 \mathrm{rpm}$, detailed view in subpanel $\mathbf{c}$; and $\mathbf{d} \omega_{\mathrm{f}}=317 \mathrm{rpm}$, detailed view in subpanel $\mathbf{e}$
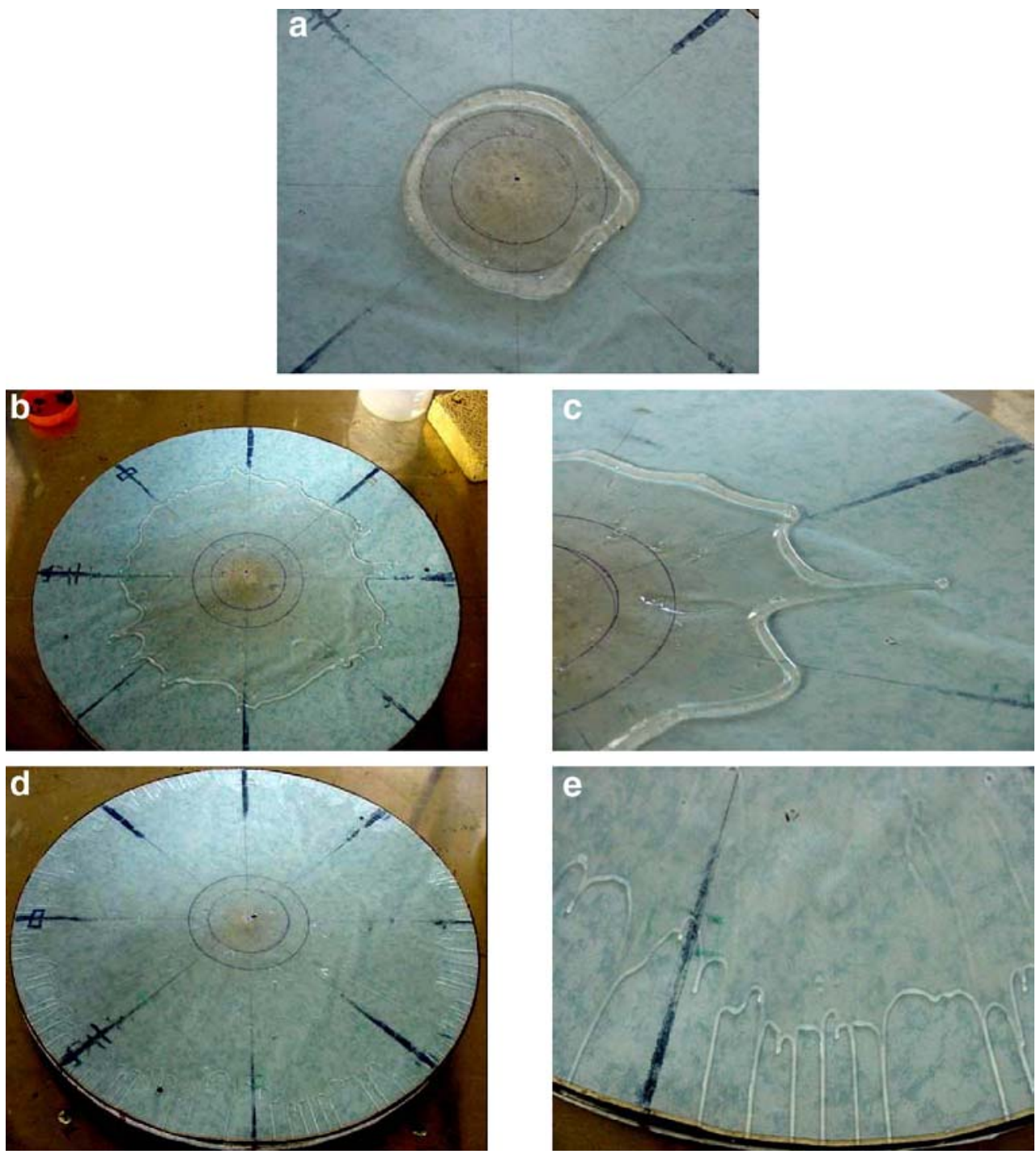

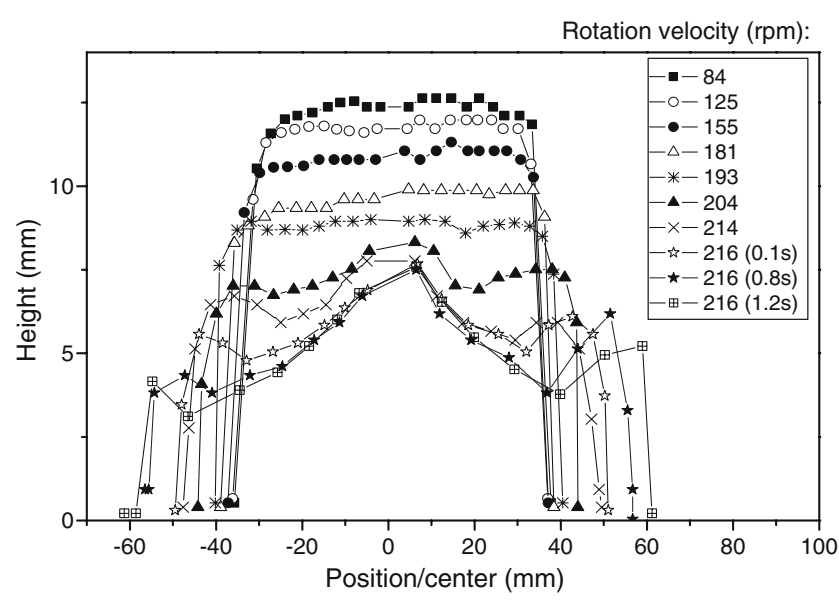

Fig. 9 Successive radial cross-section profiles of a hair gel sample in time as a function of the rotation velocity $\left(r_{0}=37.4 \mathrm{~mm}, h_{0}=13 \mathrm{~mm}\right.$, $k=7.72 \mathrm{rad} \mathrm{s}^{-2}$, and $\left.\omega_{\mathrm{f}}=216 \mathrm{rpm}\right)$. In brackets the time after the beginning of the plateau

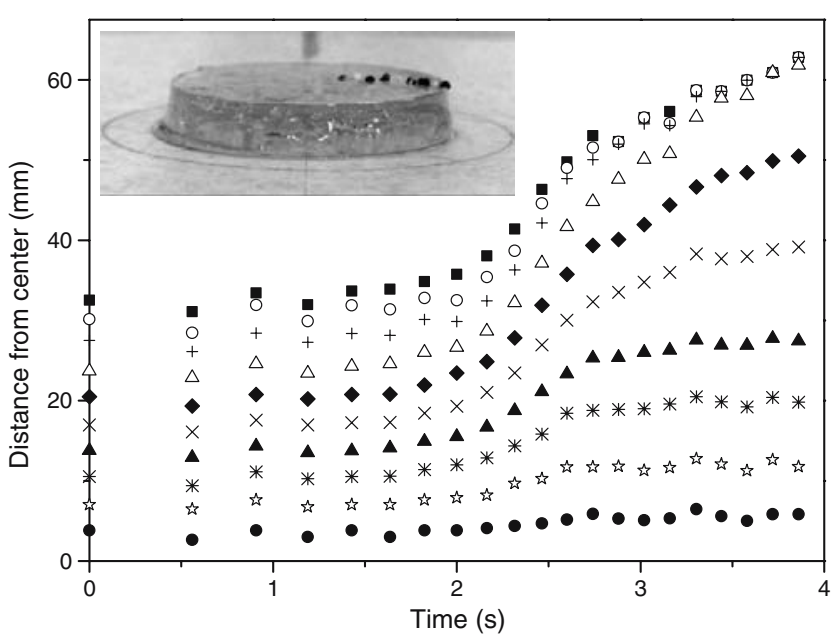

Fig. 10 Displacement of the tracers at the top of the sample during the spreading of Fig. 9. The tracers are numbered from the center to the periphery 


\section{Influence of the angular acceleration}

For different runs with the same final rotation velocity level and different accelerations $k$ (cf. Fig. 12) (for $\omega_{\mathrm{f}}>\omega_{\mathrm{c}}$ ) the curves $\langle R>$ vs $\omega$ look qualitatively similar but are shifted toward larger $\omega$ for larger $k$. All occurs as if $\omega_{\mathrm{c}}$ increases with $k$. Assuming that the critical velocity is reached when the deformation $\gamma_{0}$ is reached we deduce $\omega_{\mathrm{c}}$ in each case and rescaled the rotation velocity by $\omega_{\mathrm{c}}$. The corresponding spreading curves fall along a master curve (cf. Fig. 12). This means that the main characteristics of spreading are similar for different accelerations; only the value of the critical rotation velocity increases with $k$.

\section{Influence of the material yield stress}

We carried out tests with Carbopol 940 suspensions at different concentrations under the same acceleration. The spreading properties of these materials are similar to those obtained with the hair gel and the critical rotation velocity increases with Carbopol concentration (see Fig. 13). Moreover, after scaling the rotation velocity by $\omega_{\mathrm{c}}$, the spreading curves corresponding to different concentrations again superimpose along a master curve.

\section{Influence of the sample size}

We carried out tests with different sample radii keeping the same height. Once again we obtain similar results concerning spreading characteristics and, in particular, a master curve for the typical spreading curves as a function of the rotation velocity scaled by the critical velocity (cf. Fig. 14), but the superposition of the curves is worse than

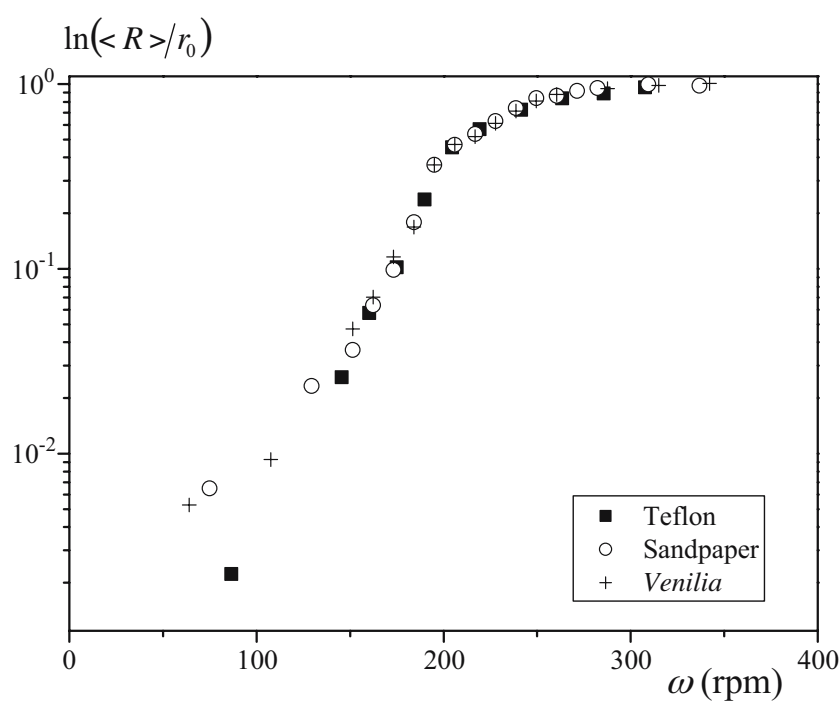

Fig. 11 Spreading as a function of rotation velocity for different solid surfaces for the hair gel $\left(r_{0}=37.4 \mathrm{~mm}, h_{0}=18 \mathrm{~mm}, k=7.72 \mathrm{rad} \mathrm{s}^{-2}\right.$, and $\left.\omega_{\mathrm{f}}=243 \mathrm{rpm}\right)$

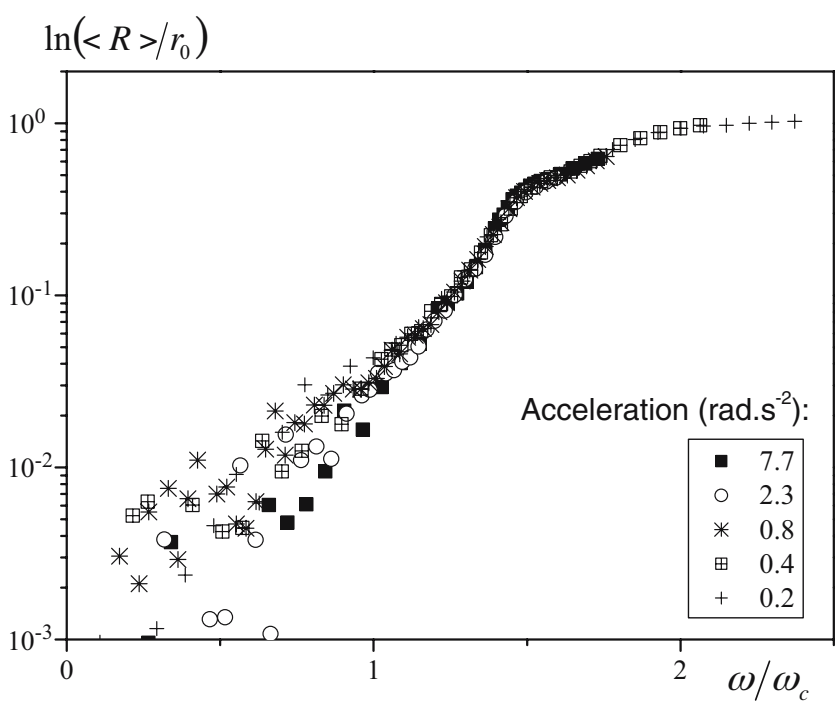

Fig. 12 Spreading as a function of dimensionless rotation velocity $(\omega /$ $\left.\omega_{\mathrm{c}}\right)$ for different accelerations for the hair gel $\left(r_{0}=37.4 \mathrm{~mm}\right.$, $h_{0}=18 \mathrm{~mm}$, and $\omega_{\mathrm{f}}=243 \mathrm{rpm}$ )

when we varied other parameters (yield stress and acceleration), especially for the largest aspect ratios $\left(h_{0} / r_{0}\right)$. The corresponding critical velocities of Figs. 12, 13, and 14 are plotted in Fig. 15.

\section{Discussion and comparison between experiments and theory}

Our observations suggest that in the first stage (regime 1) the sample undergoes a simple elongational flow, which leads to a uniform decreases of its height, as expected from the theory extrapolated to the case of $h_{0} / r_{0}$ of the order of

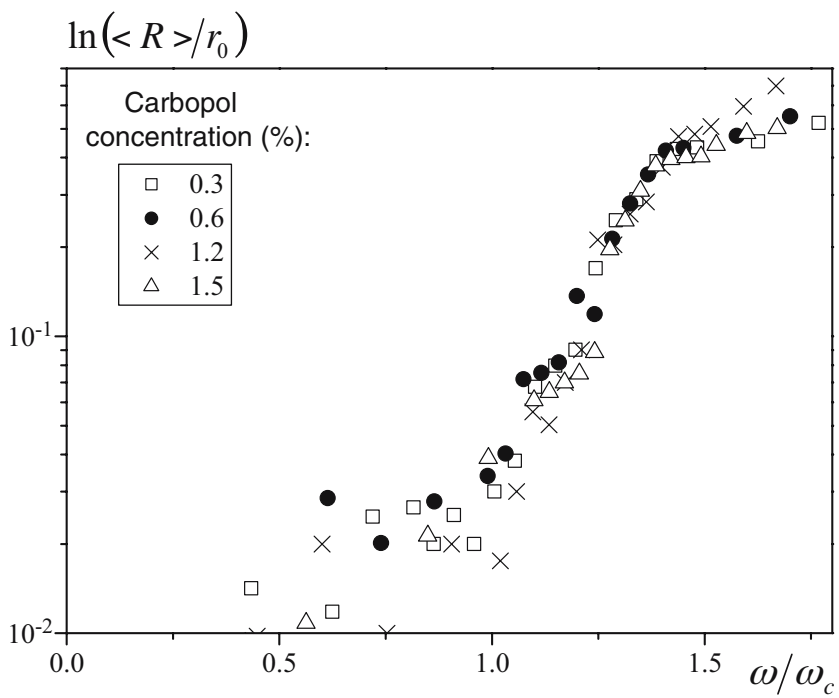

Fig. 13 Spreading as a function of dimensionless rotation velocity $(\omega /$ $\omega_{\mathrm{c}}$ ) under the same acceleration for different Carbopol concentrations $\left(r_{0}=37.4 \mathrm{~mm}, h_{0}=18 \mathrm{~mm}, k=7.72 \mathrm{rad} \mathrm{s}^{-2}\right.$, and $\left.\omega_{\mathrm{f}}=243 \mathrm{rpm}\right)$ 


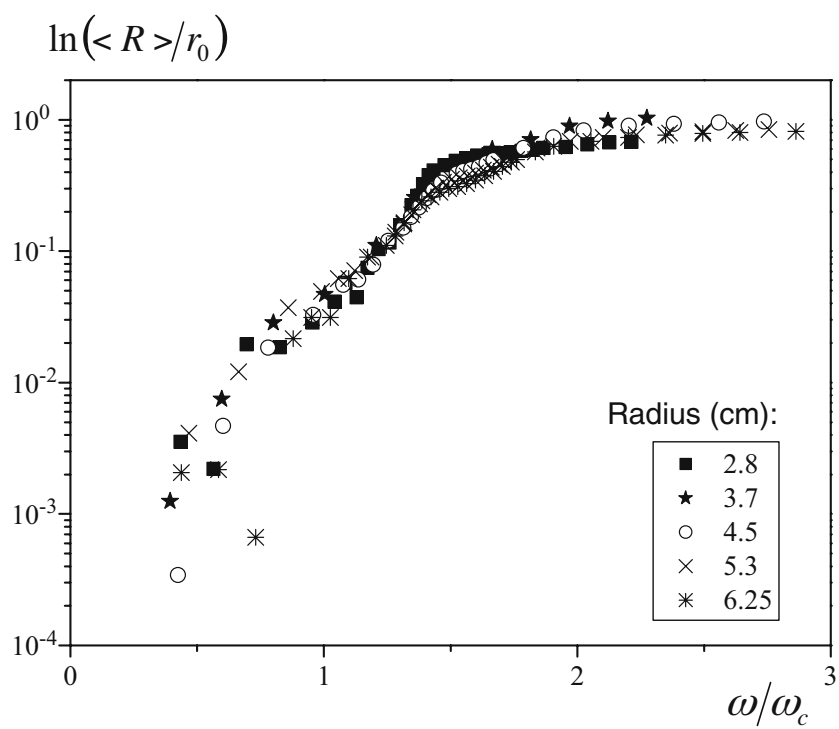

Fig. 14 Spreading as a function of dimensionless rotation velocity $(\omega /$ $\left.\omega_{\mathrm{c}}\right)$ under the same acceleration for different sample radii $\left(r_{0}=37.4 \mathrm{~mm}, h_{0}=18 \mathrm{~mm}, k=7.72 \mathrm{rad} \mathrm{s}^{-2}\right.$, and $\left.\omega_{\mathrm{f}}=243 \mathrm{rpm}\right)$

0.5 . This extrapolation seems reasonable with regard to our result, valid for any aspect ratio, that in the solid regime the flow is not influenced by gravity (see "Material setup"). Under these conditions the transition from regime 1 to regime 2 should be associated with a critical velocity $\left(\omega_{\mathrm{c}}\right)$ as given by Eq. (16). Indeed, for the hair gel we find from Baudez and Coussot (2004) that $\mu \approx 2$ Pa.s so that for our data, $t_{\mathrm{c}}$ of the order of $1 \mathrm{~s}$ is much larger than $\mu / \mathrm{G}$. However, $\omega_{\mathrm{c}}$ appears to underestimate the experimental results (see Fig. 15).

In regime 2 the fluid starts to significantly spread over the solid surface and the flow may be approximately described by the theory in the lubricational case even if $h_{0} / r_{0}$ is of the order of 0.5 at the beginning of the flow. The critical velocity $\left(\omega_{\mathrm{c}}^{*}\right)$ for flow start under such conditions is now given by Eq. (23) but this value still slightly underestimates the data (see Fig. 15). It remains that the evolutions of the theoretical critical velocity, either $\omega_{c}$ or $\omega_{\mathrm{c}}{ }^{*}$, as a function of the different parameters (yield stress and radius) are in close agreement with the data. This suggests that our theoretical approach is qualitatively valid but with a wrong coefficient somewhere, which might be due to the fact that neither the conditions for the application of the lubricational assumption or the elongational regime are perfectly valid. Moreover, although the theory predicts that the critical velocity is independent of the acceleration we find that it approximately varies as $k^{0.1}$. This might be due to the fact that viscous effects are in fact not completely negligible, in contrast with our assumptions leading to Eq. (16) or those leading to Eq. (23). Our analysis ("Solid regime") in the case of dominant viscous effects predicts a dependence of $\omega_{\mathrm{c}}=k t_{\mathrm{c}}$ as $k^{1 / 3}$, which suggests that either we $\omega_{c}(\mathrm{rpm})$

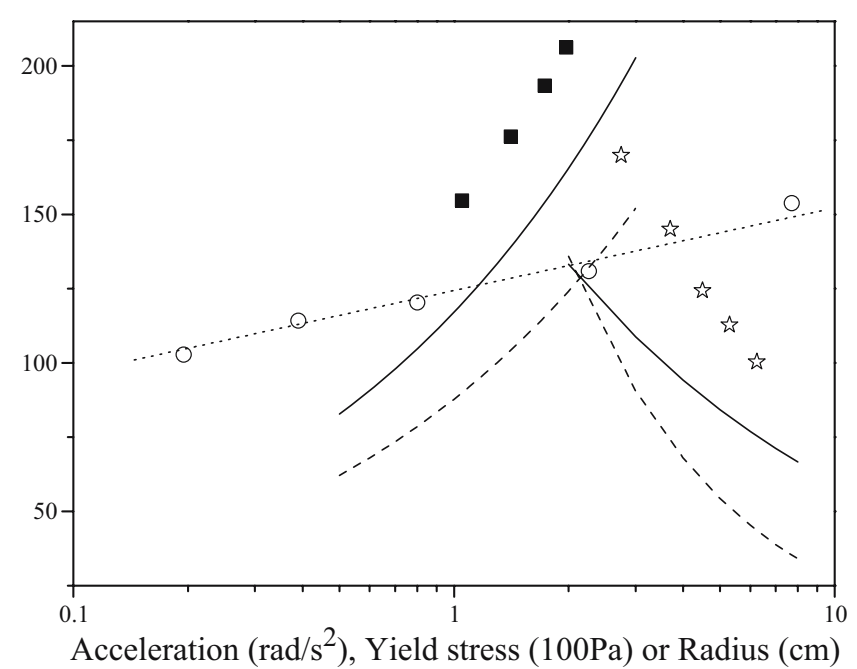

Fig. 15 Critical rotation velocity for different accelerations (spreading curves of Fig. 12) (circles), different material yield stresses (spreading curves of Fig. 13) (squares), and different sample radii (spreading curves of Fig. 14) (stars), keeping the other parameters constant. The continuous lines correspond to Eq. (23), the dashed lines to Eq. (16), and the dotted line is a power-law model of power 0.1

are in the intermediate regime or viscous effects take place in a more complex way.

The different aspects of the sample as it spreads over the disc in regimes 2 and 3 correspond well to those extrapolated from the evolution of the sample shape at equilibrium as a function of an increasing rotation velocity in the lubricational regime: first a spreading with a straight free surface at the periphery and a static region remaining in the center, then a separation between the periphery and the rest of the fluid. Moreover, for fixed initial conditions and final rotation velocity the flow properties (in terms of apparent spreading) only depend on the ratio $\omega / \bar{\omega}_{c}$, even for different material yield stresses, disc accelerations, or sample radii (see Figs. 12, 13, and 14), in agreement with the theory. This confirms that our theoretical approach provides the physical elements explaining the characteristics of the spin coating of yield stress fluids, even for $h_{0} / r_{0}$ of the order of 1 .

When the outer roll is formed the fluid further spreads mainly as a result of the motion of this roll. Indeed the centrifugal force on this roll is much larger than on the thin layer remaining behind. If the rotation velocity keeps on increasing the dome and the thin layer may nevertheless slightly spread, but this motion is negligible compared to the roll displacement. This implies that the formation of a thin layer of fluid over the disc strongly differs from a more or less uniform spreading as for a Newtonian fluid, and mainly result from the displacement and shearing of the outer roll along the solid surface, which leaves a thin layer 
of fluid behind. It is worth noting that the volume of the roll is of the order of half the total sample volume. At this stage we do not have much argument for estimating the thickness of the thin layer. We can only remark that it must be equal to or smaller than the value predicted by Eq. (23), which provides the equilibrium shape for an ideal coating (without roll). If we compute the critical thickness as predicted by Eq. (23) we find that the experimental thickness behind the roll is smaller than the theoretical thickness by a factor ranging from 1.3 to 2 . Because in this study the lubrication assumption should be valid, this discrepancy suggests that the formation of this layer is effectively governed by phenomena differing from those at the origin of the natural stoppage of a more or less uniform layer of yield stress fluid over a rotating surface.

Lastly, for sufficient large velocities the roll motion becomes unstable and fingering develops. Nevertheless, it appears difficult to compare the predictions of the existing theory (de Bruyn et al. 2002) concerning fingering of a yield stress fluid layer flowing over a solid surface with our data because the effective sample shape seems to strongly differ from that taken into account in this theory. In this study, it is clear that this is solely the motion of the roll, which must be taken into account, a motion which likely combines two important aspects: a shear flow due to the radial displacement on the disc and an tangential elongation of the roll due to the increase of its perimeter.

\section{Conclusion}

We developed a theory for the spin coating of yield stress fluids including the solid and the liquid regime of these materials, which is in rather good agreement with our experimental results. In particular we show that beyond a critical velocity the fluid separates into two parts, one at rest relatively to the plane and the other moving radially, a phenomenon which basically results from the yielding nature of such fluids. Moreover, all data in terms of deformation vs rotation velocity fall along a master curve when the rotation velocity is scaled by some critical velocity. The evolutions of the critical velocity with the yield stress, the acceleration, and the sample radius are in agreement with the theory. The absolute level of the experimental rotation velocity is nevertheless significantly larger that that predicted by theory.

The spin coating of yield stress fluids appears to fundamentally differ from that of simple liquids. Indeed, one cannot directly associate an average velocity with a fluid thickness in a stationary regime. This might become possible but only far from the pasty regime (in which the yielding plays a significant role) so that we can, as a first approximation, consider such materials as simple (unyield- ing) liquids, and most previous works in this field focused on this case. However, in contrast with previous assumptions, the spin coating of yield stress takes a very specific form: the initial solid-liquid transition followed by some spreading leads to the formation of an outer roll; the flow mainly relies on the radial motion of this roll, which leaves behind it a thin layer of fluid; lastly, fingering develops from this roll. Future work could focus on the determination of the film thickness as a function of flow characteristics and on the conditions for the development of the fingering instability.

From a general point of view this work also shows in a specific flow situation that taking into account the behavior in both the solid and the liquid regimes can be critical for describing the flow properties of yield stress fluids.

\section{References}

Acrivos A, Shah MJ, Petersen EE (1960) On the flow of a nonNewtonian liquid on a rotating disk. J Appl Phys 31:963

Balmforth NJ, Craster RV, Sassi R (2002) Shallow viscoplastic flow on an inclined plane. J Fluid Mech 470:1

Barnes HA (1997) Thixotropy—a review. J Non-Newton Fluid Mech $70: 1$

Baudez JC, Coussot P (2001) Rheology of aging, concentrated, polymeric suspensions: application to pasty sewage sludges. J Rheol 45:1123

Baudez JC, Coussot P (2004) Abrupt transition from viscoelastic solid-like to liquid-like behavior in jammed materials. Phys Rev Lett 93:128302

Baudez JC, Chabot F, Coussot P (2002) Rheological interpretation of the slump test. Appl Rheol 12:133

Bird RB, Dai GC, Yarusso BY (1982) The rheology and flow of viscoplastic materials. Rev Chem Eng 1:1

Birnie DP III (1997) Combined flow and evaporation during spin coating of complex solutions. J Non-Cryst Solids 218:174

Burgess SL, Wilson SDR (1996) Spin-coating of a viscoplastic material. Phys Fluids 8:2291

Coussot P, Proust S, Ancey C (1996) Rheological interpretation of deposits of yield stress fluids. J Non-Newton Fluid Mech 66:55

Curran SJ et al (2002) Properties of Carbopol solutions as models for yield stress fluids. J Food Sci 67:176

de Bruyn JR, Habdas P, Kim S (2002) Fingering instability of a sheet of yield stress fluid. Phys Rev E Stat Phys Plasmas Fluids Relat Interdiscip Topics 66:031504

Emslie AG, Bonner FT, Peck LG (1958) Flow of a viscous liquid on a rotating disk. J Appl Phys 29:858

Higgins BG (1986) Film flow on a rotating disk. Phys Fluids 29:3522

Huang YY, Chou KS (2003) Studies of the spin coating process of silica films. Ceram Int 29:485

Jenekhe SA, Schuldt SB (1984) Coating flow of non-Newtonian fluids on a flat rotating disk. Ind Eng Chem Fundam 23:432

Jenekhe SA, Schuldt SB (1985) Flow and film thickness of Bingham plastic liquids on a rotating disk. Chem Eng Commun 33:135

Lawrence CJ, Zhou W (1991) Spin coating of non-Newtonian fluids. J Non-Newton Fluid Mech 39:137

Lipscomb GG, Denn MM (1984) Flow of Bingham fluids in complex geometries. J Non-Newton Fluid Mech 14:337

Liu FK, Mei CC (1989) Slow spreading of a Bingham fluid on an inclined plane. J Fluid Mech 207:505 
Oldroyd JG (1947) A rational formulation of the equations of plastic flow for a Bingham solid. Proc Camb Philos Soc 43:100

Pashias N, Boger DV, Summers J, Glenister DJ (1996) A fifty cent rheometer for yield stress measurements. J Rheol 40:1179

Thirion F, Chabot F (2003) Spreading of sewage sludge: machines and practices. Cemagref Editions, Paris
Tsamopoulos JA, Chen MF, Borkar AV (1996) On the spin coating of viscoplastic fluids. Rheol Acta 35:597

Uhlherr PHT, Guo J, Tiu C, Zhang XM, Zhou JZQ, Fang TN (2005) The shear-induced solid-liquid transition in yield stress materials with chemically different structures. J Non-Newton Fluid Mech 125:101 